\title{
Rare e14a3 (b3a3) BCR-ABL fusion in chronic myeloid leukemia in India: The threats and challenges in monitoring minimal residual disease (MRD)
}

\author{
Salil Vaniawala ${ }^{\mathrm{a}}$, Arpan Acharya ${ }^{\mathrm{b}}$, Harsh Parekh $^{\mathrm{c}}$ and Pratap N. Mukhopadhyaya ${ }^{\mathrm{c}, *}$ \\ ${ }^{a} S N$ Genelab, Surat, Gujarat, India \\ ${ }^{\mathrm{b}}$ Dr. D.Y. Patil Biotechnology \& Bioinformatics Institute, Tathawade, Pune, India \\ ${ }^{c}$ Interdisciplinary Science, Technology and Research Academy, AISC, New Modhikana, Katad Khana, Pune, India
}

\begin{abstract}
.
OBJECTIVE: The primary objective of this work was to confirm the occurrence of rare $B C R A B L$ fusion variant involving the a3 region of the $A B L$ gene in a patient positive for $\mathrm{t}(9 ; 22)$ translocation but negative for common major and minor breakpoint cluster regions and the challenges and threats that it poses in a routine laboratory setting which use commercial kits for monitoring the minimal residual disease.

METHODS: A patient with elevated white blood cell count was subjected to classical cytogenetics, FISH as well as RT-PCR testing using commercial kits as well as published primers and in house testing protocol. PCR amplicon generated from in the process was sequenced and analyzed.

RESULTS: The translocation event in chromosome 9 and 22 could be successfully detected. BCR/ABL dual color, dual fusion probe generated a classical balanced translocation scenario within the nucleus of affected cells and presented a ' $1 \mathrm{O} 1 \mathrm{G} 2 \mathrm{~F}$ ' signal pattern. RT-PCR with probes from commercial kit designed to detect common breakpoints within the M- and m-BCR regions involving e13a2, e14a2 and e1 a 2 fusion variants respectively failed to generate any signal. Further investigation revealed presence of the rare e14a3 (b3a3) fusion.

DISCUSSION: This is the first report of rare e14a3 fusion in the BCR ABL gene in a CML patient from India. The observation indicates the need for interrogating rare BCR ABL fusions when common breakpoint cluster regions are absent such that minimal residual disease (MRD), critical for disease monitoring, can be performed and false positive remission cases can be avoided. It also emphasizes the utility and significance of cytogenetics and FISH techniques in primary diagnosis of CML and use of RT-PCR based assays only for generating secondary information within special reference to MRD.

CONCLUSION: The rare e14a3 (b3a3) fusion of the $B C R A B L$ gene is present in Indian population as demonstrated from this first report and clinical laboratories using commercial kit that do not cover such rare fusions are likely to generate false result thereby declaring complete molecular remission in CML patients under therapy while conducting MRD assay using RT-PCR technology.
\end{abstract}

Keywords: CML, Leukemia, breakpoint, b3a3, e14a3

*Corresponding author: Dr. Pratap N. Mukhopadhyaya, Interdisciplinary Science, Technology and Research Academy, AISC, 2390-B, K.B. Hidayatullah Road, New Modhikana, Katad Khana, Pune, India. Tel.: +91 9881153425; E-mail: pnm.istra@yahoo.com. 


\section{Introduction}

More than $90 \%$ of all Chronic Myeloid Leukemia (CML) [1] and around 10-25\% of acute lymphoblastic leukemia (ALL) cases [2] are associated with a translocation between chromosome 9 and 22. This results in birth of a new hybrid $B C R A B L$ gene on the translocation-derived Philadelphia chromosome. The breaks on chromosome 22q11.2 generally take place in the major $(\mathrm{M}-b c r)$ and minor $(\mathrm{m}-b c r)$ breakpoint cluster region or (rarely) in the neighboring sites. On the other hand, the breaks in chromosome 9q34 involve the $A B L$ gene, the name being derived from the Abelson murine leukemia virus where this category of gene was first reported. This fusion of part of the BCR and the ABL genes brings forth the $5^{\prime}$ end of the former into juxtaposition with tyrosine kinase domain of the later [3] retaining the phosphorylation activity of the ABL gene. Depending on whether the breakpoint is major $(\mathrm{M}-B C R)$ or minor $(\mathrm{m}-B C R)$, the transcription of this newly formed fusion gene results in mRNA coding for either $210 \mathrm{Kd}$ or $190 \mathrm{Kd}$ protein respectively. This fusion protein has immense pathological significance and is unequivocally associated with conditions of CML.

Detection of $\mathrm{t}(9 ; 22)$ translocation is important in cases of CML and karyotyping technique is one of the robust assays prescribed even today for newly diagnosed leukemia patients. The method has advantage of very high specificity and can detect additional chromosomal aberrations also since the entire set of chromosomes comes under the analysis purview. However, it is worthwhile to state that this method requires viable marrow cells or greater than $10 \%$ blasts to culture the cells and detect metaphase conditions. Hindrances include occasional fibrosis that interferes in the process of aspiration of marrows which in turn lead to lesser number of metaphase cells [4].

Fluorescent in situ hybridization offers a wider choice of detection methods including the option of identifying $B C R A B L$ translocation in either metaphase or interphase cells. Since interphase cells are also suitable, this technique can be applied to blood leukocytes and other non-dividing cells. It can also detect complex $B C R A B L$ rearrangements such as three-way translocation or breaks outside the minor and major cluster regions [4].

With time, reverse transcription polymerase chain reaction (RT-PCR) has evolved as a sensitive method for detecting $B C R A B L$ translocation. One of the promi- nent features of this technique is its ability to target messenger RNA population rather than genomic DNA. The later is far more homogenous in nature and allows PCR primers to anneal at shorter distances from each other compared to corresponding location within the genomic DNA [5].

CML is one of the first and perhaps the best success stories for allogenic tranaplantation and it is in this context that the necessity of molecular monitoring came into prominence in recent times. Commonly known as determination of the minimal residual disease or MRD, this is done by the method of RT-PCR which is found to be useful in predicting future relapses [6-8]. The advent of tyrosine kinase inhibitors (TKIs) further enhanced the significance and importance of RT-PCR-based detection of MRD in CML patients since it assists in providing the much-needed quantitative estimate of efficacy of a drug in the patient.

The rapid success of RT PCR based detection of MRD is primarily based on accurate understanding of the breakpoint regions that are involved in birth of the chimerical BCR-ABL gene apart from the revolutionary discovery of reverse transcription and PCR technology [9]. This in turn led to determination of the exact hybridization location of the synthetic probes, crucial for the assay.

Designing of RT-PCR primers rely heavily on the precise location of the breakpoints associated with $\mathrm{t}(9 ; 22)$ translocation and formation of the $B C R-A B L$ gene. In CML, over $95 \%$ of the breakpoints involving the M- $b c r$ region comprise of those downstream of either exon 13 (e13; earlier known as b2) or 14 (e14; earlier known as b3) and that of the $A B L$ gene upstream of exon 2 (a2). In other words the e1- e13 or e1- e14 region of the BCR gene fuses with a 2 - a11 region of the $A B L$ gene to give rise to the chimerical gene coding for a $210 \mathrm{Kd}$ protein with pathological tyrosine kinase activity [10]. Two less common translocations are also known between $B C R$ exon 2 (m-bcr) and exons 19/20 $(\mu$-bcr) which code for a $190 \mathrm{Kd}(\mathrm{e} 1 \mathrm{a} 2)$ and $230 \mathrm{Kd}$ (e19a2) proteins respectively $[11,12]$.

Apart from these, some rare breakpoints are also reported which may be broadly categorized into 4 groups. $B C R$ breakpoints located within introns that exist outside M-bcr, m-bcr \& $\mu$-bcr and fused to $A B L$ a2; $B C R$ breakpoints that occur within its exons and fused to $A B L$ a2; M-bcr, m-bcr \& $\mu$-bcr breakpoints fused to $A B L$ gene downstream of a 2 and transcripts comprising of intervening sequences between $B C R$ and $A B L$ a2 genes [13]. 
The high degree of sensitivity by way of detection of around $0.01 \%$ or fewer BCR-ABL fusion transcript now place quantitative RT-PCR test in the list of standard protocols for estimating MRD of patients undergoing Tyrosine kinase inhibitor (TKI) treatment as laid down in the 2009 National Comprehensive Cancer Network clinical practice guidelines for CML (Minimal Residual Disease MRD-A Participant Summary: College of American Pathologists, 2008, p 17). Therefore it is necessary to maintain a strict vigil on the efficacy of commonly used RT-PCR tests with special reference to the hybridization locations of the PCR oligonucleotide primers such that false negativity is avoided even if a certain version of breakpoint is less common in the population.

In this study we investigated a patient with $\mathrm{t}(9 ; 22)$ translocation and encountered a rare variant of $B C R$ $A B L$ gene fusion which require a different protocol to be followed for estimating MRD. To our knowledge, this is the first report of a specific rare $B C R-A B L$ fusion variant from India.

\section{Materials and methods}

Before initiation of the study written consent was taken from the concerned patient. The study was duly approved by bio-safety and ethics committee of our institute (SNGL/2013/24W). Blood and bone marrow samples were collected in $\mathrm{K}_{2}$-EDTA and sodium heparin vacutainer tubes (Becton Dickinson, san Diego, Calif) for molecular and cytogenetic studies respectively.

\subsection{Cytogenetics study and fluorescent in situ hybridization analysis}

Bone marrow cells from the patient were cultured in vitro according to standard laboratory protocol and 30 G-banded metaphases and two karyotypes were prepared and analyzed respectively. FISH study was performed using the LSI BCR/ABL dual color, dual fusion probe set (Abbott Molecular Inc., Des Plaines, IL). A normal nucleus showed two separate orange and green signals $(2 \mathrm{O} 2 \mathrm{G})$ while a nucleus bearing reciprocal translocation $[\mathrm{t}(9 ; 22)]$ showed one orange, one green and two orange/green fusion signals $(1 \mathrm{O} 1 \mathrm{G} 2 \mathrm{~F})$. Around 200 interphase cells were screened for the patient and subsequently analyzed by two independent researchers.

\subsection{Reverse Transcription PCR for detecting $B C R-A B L$ breakpoint}

Total RNA was isolated from patient sample (peripheral blood and bone marrow) using QIAamp RNA Mini blood kit (Qiagen Inc., Valencia, CA) and quantified by spectrophotometric analysis at 260 and $280 \mathrm{~nm}$.

\subsection{Breakpoint b2a2, b3a2 \& ela2}

Routine BCR-ABL transcript detection was performed using the BCR-ABL Mbcr/ABL FusionQuant kit (Ipsogen, New Haven, CT) as per manufacturer's protocol. This kit allows amplification of BCR-ABL and $A B L$ transcripts in two independent reactions. The $\mathrm{M}-\mathrm{BCR}$ and $\mathrm{m}-\mathrm{BCR}$ regions are targeted and the breakpoints covered are e13a2 (b2a2) and e14a2 (b3a2) in the M-BCR while e1a2 in the $\mathrm{m}-\mathrm{BCR}$ region.

\subsection{Breakpoint e14a3}

For detecting the rare transcript with e $14 \mathrm{a} 3$ breakpoint, cDNA synthesis was performed using $1 \mu \mathrm{g}$ of extracted RNA isolated from the bone marrow specimen using the PrimeScript 1st strand cDNA synthesis kit (TaKaRa-bio, CA, USA) according to the manufacturer's protocol. Thermal amplification of the region harboring the e14a3 breakpoint was performed as described by Dongen et al., 1999. Briefly, the PCR reactions contained $1 \mathrm{X}$ PCR buffer, $1.5 \mathrm{mM} \mathrm{MgCl}_{2}$, $200 \mathrm{mM}$ (each) deoxynucleotide triphosphates, 10 pmoles of forward and reverse primers and 1.0 unit of Taq DNA polymerase (Life Technologies, USA) in a $25 \mu \mathrm{l}$ reaction using a Veriti 96 well thermal cycler (Applied Biosystems; USA). The thermal cycling condition was as follows: $95^{\circ} \mathrm{C}$ for $2 \mathrm{~min}$ followed by $95^{\circ} \mathrm{C}$ for $30 \mathrm{sec}, 65^{\circ} \mathrm{C}$ for $30 \mathrm{sec}$ and $72^{\circ} \mathrm{C}$ for $40 \mathrm{sec}$ for a total of 35 cycles and a $5 \mathrm{~min}$ final extension at $72^{\circ} \mathrm{C}$. PCR products were resolved on a $2 \%$ agarose gel (Promega Corporation, Madison, USA), stained with ethidium bromide $(0.5 \mu \mathrm{g} / \mathrm{ml})$ and image captured using a Bio Rad gel documentation system (BIORAD, USA).

PCR amplicon was purified using QIAGEN PCR purification kit (QIAGEN, Germany), and the purified product was subjected to double stranded fluorescent nucleotide sequencing using the BigDye Terminator v3.1 Cycle Sequencing Kit and an ABI PRISM ${ }^{\circledR} 3500$ 
Dx Genetic Analyzer platform (Applied Biosystems, Calif) as per manufacturer's instructions.

\subsection{Case report}

A 30 year old patient with persistently elevated white blood cell count (WBC) was referred to our clinical laboratory for cytogenetics investigation related to condition of CML. His peripheral blood differential was left-shifted but presented no peripheral blasts. The peripheral blood smear too did not present evidence of acute leukemia or dysplasia although the white blood cell count remained elevated. At this point blood was taken for detection of Philadelphia chromosome $(\mathrm{Ph})$ by cytogenetics and also for BCR-ABL rearrangement by RT-PCR. At this stage we found the sample to be positive for $\mathrm{Ph}$ chromosome but consistently negative for the most common rearrangements associated with $\mathrm{M}-\mathrm{BCR}$ and $\mathrm{m}-\mathrm{BCR}$ respectively. We thereafter proceeded to undertake FISH analysis and also investigate for other $\mathrm{BCR} \mathrm{ABL}$ rearrangements using different primer pairs.

\section{Results and discussion}

Following proper study of the medical history of the patient a repeat bone marrow aspiration and biopsy with complete morphological, cytological and molecular testing was performed. Bone marrow biopsy test indicated a markedly hyper-cellular marrow with myeloid predominance. Cytogenetic analysis presented a karyotype of 46, XY, t(9;22)(q34;q11.2) [25/25] (Fig. 1). FISH analysis confirmed BCR-ABL translocation with $>90 \%$ of cells showing balanced translocation between chromosome 9 and 22 and with 1O1G2F pattern (Fig. 2). White blood cell count of peripheral blood sample was also found to be approximately $45,000 / \mathrm{mm}^{3}$ and demonstrating $90 \%$ BCR-ABL FISH positivity.
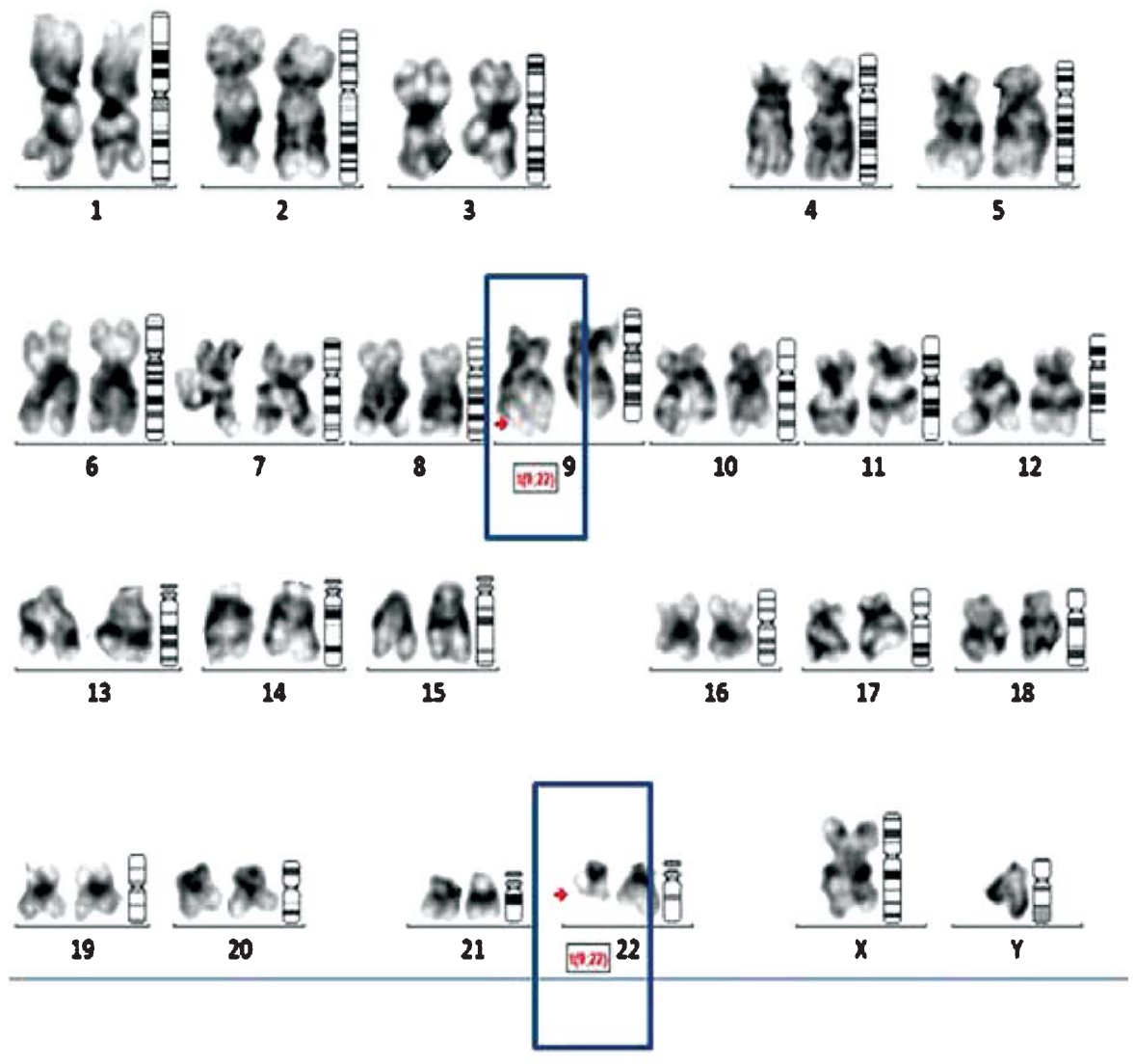

Fig. 1. Cytogenetic analysis showing chromosomal translocation $[\mathrm{t}(9 ; 22)]$ in chromosome 9 and 22 (black boxes). 


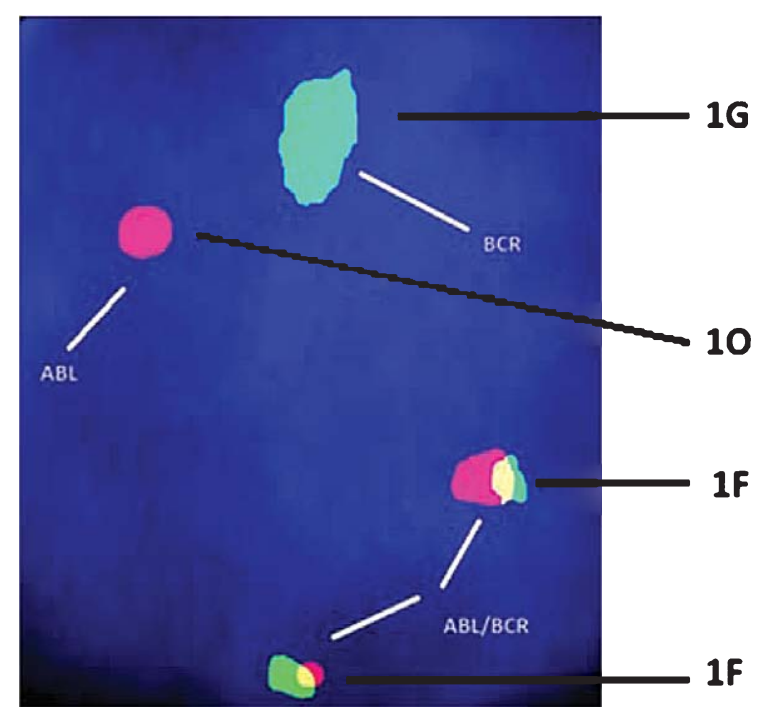

Fig. 2. FISH analysis using LSI BCR/ABL dual color, dual fusion probe set (Abbott Molecular Inc., Des Plaines, IL). A nucleus showing reciprocal translocation $[\mathrm{t}(9 ; 22)]$ with ' $1 \mathrm{O} 1 \mathrm{G} 2 \mathrm{~F}$ ' pattern. 1G: 1 green signal; 1O: 1 orange signal; $1 \mathrm{~F}$ : 1 fusion signal showing both orange and green color.

RT PCR was performed to target three most common BCR ABL breakpoints. These were b2a2 (e13a2) and b3a2 (e14a2) representing the major breakpoint cluster region (M-BCR) and e1a2 from the minor breakpoint cluster region (m-BCR). Repeat RT-PCR tests for all these three loci were found to be negative (Fig. 3). In view of the persistent and confirmed presence of miniature $\mathrm{Ph}$ chromosome detected in cytogenetic test and the $1 \mathrm{O} 1 \mathrm{G} 2 \mathrm{~F}$ pattern in FISH coupled with absence of common BCR-ABL breakpoints, we proceeded to test for the presence of rare variants of BCR-ABL gene fusion.

Thermal amplification of the e14a3-harboring BCRABL hybrid cDNA with the forward primer annealing to BCR (NM_021574; nucleotide position 3194-3215) and the reverse primer annealing to ABL (NM_005157' nucleotide position 314-294) region (Fig. 4A \& B) as described by Dongen et al. (1999) generated a 243 bp PCR amplicon (Fig. 4C). Since interrogation of the breakpoint cannot be detected by classical methods of identifying DNA mutation such as PCR-RFLP, we sequenced the amplicon (Fig. 4D) using both the forward and the reverse primers. Results indicated that the break occurred in nucleotide position 3378 of the BCR and 257 of the ABL gene (Fig. 4E) making this case a rare e $14 \mathrm{a} 3$ category of BCR ABL translocation.

The $\mathrm{p} 210$ and p190 protein-coding transcripts from $\mathrm{b} 2 \mathrm{a} 2, \mathrm{~b} 3 \mathrm{a} 2$ and e1a2 rearrangements respectively dominate the clinical testing services in developing countries. The other less common variants that generate transcripts from $\mathrm{b} 3 \mathrm{a} 3$ or $\mathrm{b} 2 \mathrm{a} 3$ (p203 protein) and e19a2 (p230 protein) [14] are rare and often not covered in routine screening and analysis. Fusion transcripts involving a3 region of ABL gene such as one reported in this study (e14a3 or b3a3) needs a special mention. The BCR ABL a3 transcript does not have part of the ABL SH3 domain which is present in all other transcripts involving a2 part of the ABL gene. This SH3 domain induces leukemogenesis by downregulating the kinase domain (SH1) and activating the STAT5 signaling pathway $[15,16]$. Hence it is theoretically expected that the clinical expression of a3 variants of CML would be different than those associated with a2 ones. Interestingly, this ABL a3 breakpoint does not affect the sequence associated with ATP / imatinib mesylate-binding domain of the kinase although alteration in the tertiary structure and subsequent effect on drug response cannot be ruled out. At least 3 reported cases involving this category of breakpoint within the BCR ABL gene were found to respond well to imatinib mesylate and indicated classical disease progression [17].

According to a report, around 9 cases (10 including the one reported in this study) of BCR ABL translocations involving a3 region of the $\mathrm{ABL}$ gene has been reported in the world [18] and $>90 \%$ of the patients 

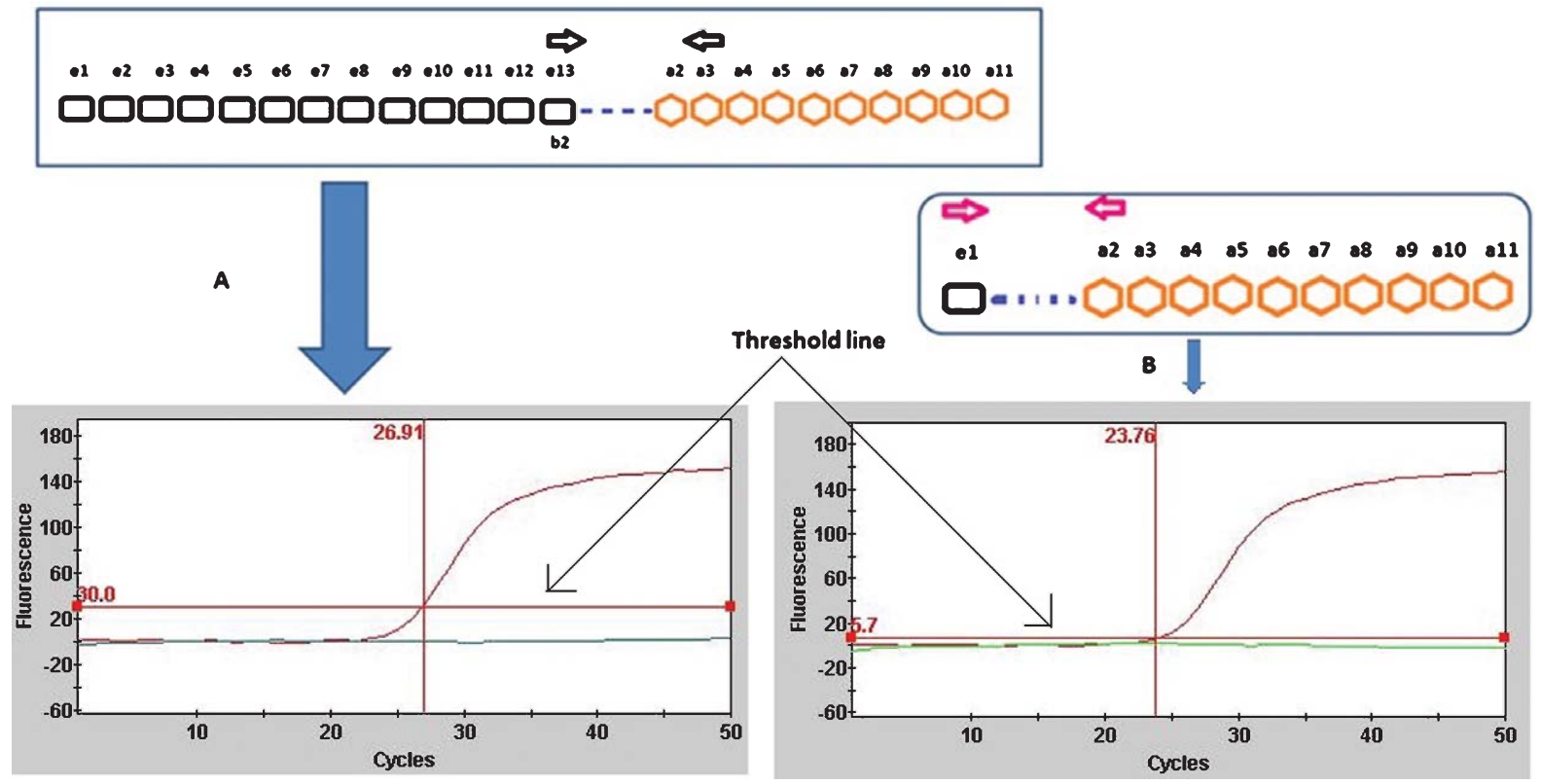

Fig. 3. Reverse Transcription - Real Time PCR qualitative analysis of the clinical sample using two primer sets targeting the common BCR-ABL breakpoints. A: PCR probes that detect e13a2 (b2a2) and e14a2 (b3a2) rearrangements within the Major Breakpoint Cluster Region (M-BCR). The corresponding real time PCR result show target signal below the threshold line while positive control (ABL gene target) generating a 'S' curve with Ct value between 20-30. B: PCR probe designed to detect ela2 breakpoint within the minor Breakpoint Cluster Region. The corresponding real time PCR result is similar to that shown for ' $\mathrm{A}$ ' with the target signal running below threshold line while the positive control (ABL gene target) showing a $\mathrm{Ct}$ value between 20-25. In both 'A' and 'B', the signal is negative indicating that the translocation breakpoints targeted in both these reactions were not present.

were below the age of $40[19,20]$. Given the fact that our patient was also aged 30 years, it may be possible that this specific breakpoint is associated with younger patients. Further, such patients were also associated with very high WBC count and demonstrated progression of the disease during a-IFN therapy [18]. Given the fact that in our case we observed high WBC count, it warrants further study to know whether this feature of disease progression during a-IFN therapy is specific for a3-ABL category of translocation breakpoints.

In a study by College of American Pathology in the year 2008, almost half of 82 different clinical laboratories used commercial kit for conducing minimal residual disease of $B C R A B L$ in CML patients [14]. The commercial kit market is dominated Ipsogen (New Haven, CT) and Cepheid (Sunnyvale, CA) and both these assays use primer sets located in exon 13 of BCR and exon 2 of ABL, which allow for detection of common e13a2 and e14a2 translocations (M-bcr, p210) but fail to amplify the rare variants lacking $A B L$ exon 2 (BCR-ABL a3 type transcripts). In such a scenario, rare $\mathrm{BCR} A B L$ fusants such as the one reported in this study are likely to generate false negative results giving the impression of complete molecular remission (CMR) [21].

To conclude, we report here the first CML case from India involving e14a3 (b3a3) breakpoint within the BCR ABL gene. This is a challenging scenario since classical quantitative (q) or qualitative RT-PCR tests [22] do not cover this rare breakpoint and is likely to generate false negative results and an impression of CMR. The National Comprehensive Cancer Network (NCCN) Practice Guidelines (v2. 2009) profess use of cytogenetics, FISH and qRT-PCR for addressing chronic phase of this disease. CML in adults should ideally bypass this problem since both cytogenetics as well as FISH effectively identify the translocation though not the specific breakpoints as was effectively shown in our study. But qRT-PCR based monitoring of the disease every 3-6 months as stipulated in NCCN guidelines will be difficult using standard commercial kits that does not allow screening of rare $B C R A B L$ fusants. These important points coupled with the probable variation in disease progression and drug response 


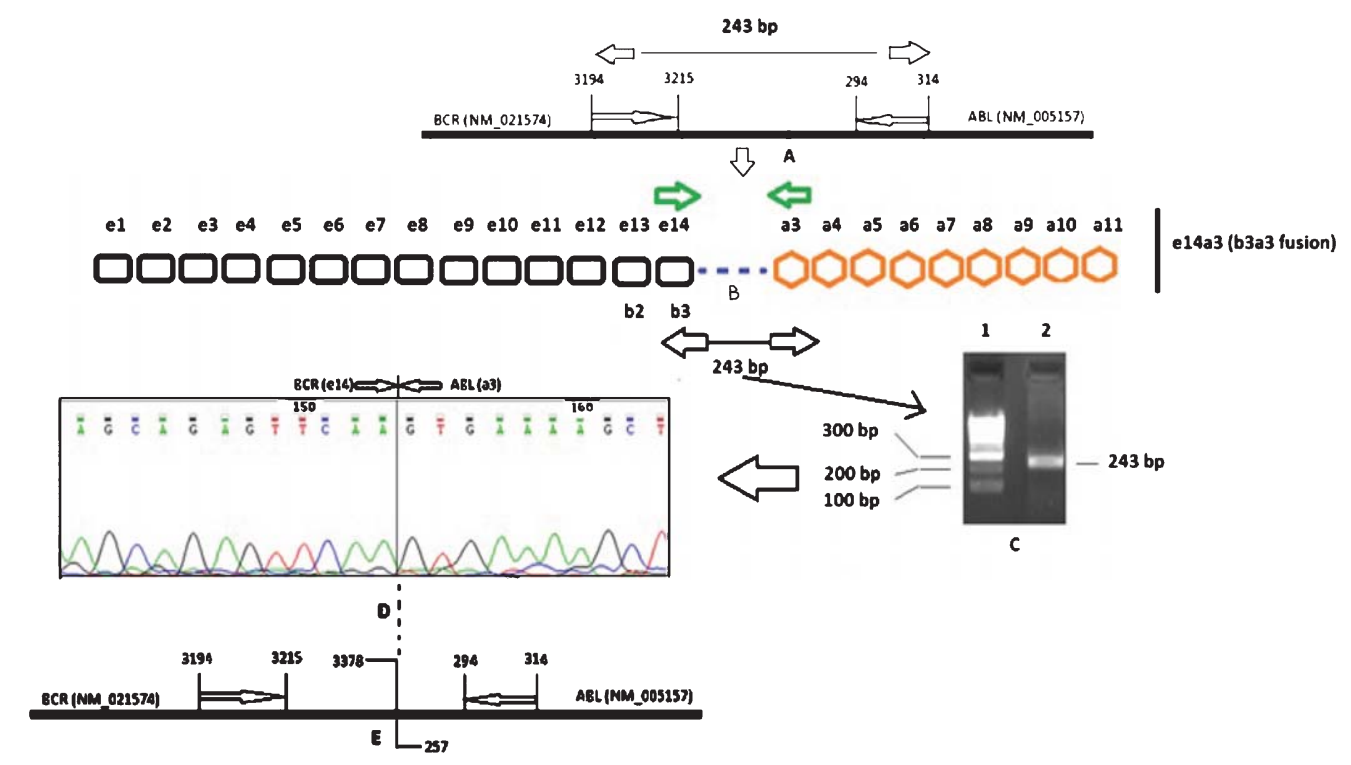

Fig. 4. Detection of a rare breakpoint (e14a3 or b3a3) from cDNA generated from a clinical sample showing $\mathrm{t}(9 ; 22)$ translocation. A: Coordinates of the BCR and the ABL gene where the forward and the reverse primers hybridize respectively to generate the $243 \mathrm{bp} \mathrm{PCR}$ amplicon. B: Exonic regions of the BCR and the ABL genes that were amplified for detection of the e14a3 breakpoint. C: A 243 bp PCR amplicon generated that contain the rare e $14 \mathrm{a} 3$ breakpoint. D: Electropherogram showing the exact point of fusion of the BCR and ABL genes to generate the e14a3 breakpoint. E: Schematic diagram showing nucleotide positions of the BCR and the ABL genes respectively where break occurred to generate the $14 \mathrm{a} 3$ breakpoint.

that might be associated with such rare breakpoints suggest that further study and screening is necessary in the population for detecting such rare breakpoints. It is also necessary to estimate the trend of their horizontal and vertical spread in the population irrespective of their incidence rate and geographical distribution.

\section{References}

[1] J.E. Cortes, M. Talpaz, M. Beran, S.M. O'Brien, M.B. Rios, S Stass and H.M. Kantarjian, Philadelphia chromosome-negative chronic myelogenous leukemia with rearrangement of the breakpoint cluster region. Long-term follow-up results, Cancer 75 (1995), 464-470.

[2] S.S. Clark, J. McLaughlin, M. Timmons, A. Pendergast, Y. Ben Neriah, L.W. Dow, W. Crist, G. Rovera, S.D. Smith and O.N. Witte, Expression of a distinctive BCR-ABL oncogene in Ph1-positive acute lymphocytic leukemia (ALL), Science 239 (1988), 775-777.

[3] R.L. Davis, J.B. Konopka and O.N. Witte, Activation of the c-abl oncogene by viral transduction or chromosomal translocation generates altered c-abl proteins with similar in vitro kinase properties, Mol Cell Biol 5 (1985), 204-213.

[4] A.F. Pelz, H. Kron̈ing, A. Franke, P. Wieacker and M. Stumm, High reliability and sensitivity of the bcr/ABL1 D-FISH test for the detection of BCR/ABL rearrangements, Ann Hematol 81 (2002), 147-153.
[5] J.Y. Sidorova, L.B. Saltykova, A.A. Lyschov, A.Y. Zaritskey, K.M. Abdulkadyrov and M.N. Blinov, A rapid RT-PCR based method for the detection of BCR-ABL translocation, Mol Pathol 50 (1997), 266-268.

[6] T.P. Hughes, G.J. Morgan, P. Martiat and J.M. Goldman, Detection of residual leukemia after bone marrow transplant for chronic myeloid leukemia: Role of polymerase chain reaction in predicting relapse, Blood 77 (1991), 874-878.

[7] T. Lion, T. Henn, A. Gaiger, P. Kalhs and H. Gadner, Early detection of relapse after bone marrow transplantation in patients with chronic myelogenous leukaemia, Lancet 341 (1993), 275-276.

[8] J.P. Radich, G. Gehly, T. Gooley, E. Bryant, R.A. Clift, S. Collins, S. Edmands, J. Kirk, A. Lee and P. Kessler, Polymerase chain reaction detection of the BCR-ABL fusion transcript after allogeneic marrow transplantation for chronic myeloid leukemia: Results and implications in 346 patients, Blood 85 (1995), 2632-2638.

[9] A.L. Nashed, K.W. Rao and M.L. Gulley, Clinical applications of BCR-ABL molecular testing in acute leukemia, Journal of Molecular Diagnostics 5 (2003), 63-72.

[10] S.L. Chissoe, A. Bodenteich, Y.F. Wang, Y.P. Wang, D. Burian, S,W. Clifton, J. Crabtree, A. Freeman, K. Iyer and J. Jian, Sequence and analysis of the human ABL gene, the BCR gene, and regions involved in the Philadelphia chromosomal translocation, Genomics 27 (1995), 67-82.

[11] F. Pane, F. Frigeri, M. Sindona, L. Luciano, F. Ferrara, R. Cimino, G. Meloni, G. Saglio, F. Salvatore and B. Rotoli, Neutrophilic-chronic myeloid leukemia: A distinct disease 
with a specific molecular marker (BCR/ABL with C3/A2 junction), Blood 88 (1996), 2410-2414.

[12] F. Ravandi, J. Cortes, M. Albitar, R. Arlinghaus, G.J. Qiang, M. Talpaz and H.M. Kantarjian, Chronic myelogenous leukemia with p185 (BCR/ABL) expression: Characteristics and clinical significance, Br J Haematol 107 (1999), 581-586.

[13] D.J. Barnes and J.V. Melo, Cytogenetic and molecular genetic aspects of chronic myeloid leukemia, Acta Haematologica 108 (2002), 180-202.

[14] N. Jinawath, A. Norris-Kirby, B.D. Smith, C.D. Gocke, D.A. Batista, C.A. Griffin and K.M. Murphy, Consultations in molecular diagnostics a rare e14a3 (b3a3) BCR-ABL fusion transcript in chronic myeloid leukemia. Diagnostic challenges in clinical laboratory practice, Journal of Molecular Diagnostics 11 (2009), 359-363.

[15] T. Skorski, M. Nieborowska-Skorska, P. Wlodarski, M. Wasik, R. Trotta, P. Kanakaraj, P. Salomoni, M. Antonyak, R. Martinez, M. Majewski, A. Wong, B. Perussia and B. Calabretta, The SH3 domain contributes to BCR/ABL-dependent leukemogenesis in vivo: Role in adhesion, invasion, and homing, Blood 91 (1998), 406-418.

[16] M. Nieborowska-Skorska, M.A. Wasik, A. Slupianek, P. Salomoni, T. Kitamura, B. Calabretta and T. Skorski, Signal transducer and activator of transcription (STAT)5 activation by BCR/ABL is dependent on intact Src homology (SH)3 and $\mathrm{SH} 2$ domains of $\mathrm{BCR} / \mathrm{ABL}$ and is required for leukemogenesis, J Exp Med 189 (1999), 1229-1242.
[17] S. Fujisawa, S. Nakamura, K. Naito, M. Kobayashi and K. Ohnishi, A variant transcript, e1a3, of the minor BCR-ABL fusion gene in acute lymphoblastic leukemia: Case report and review of literature, Int J Hematol 87 (2008), 184-188.

[18] M. Amabile, G. Martinelli, C. Terragna, V. Montefusco, A. Tabilio and S. Tura, An atypical (b3/a3) junction of the bcr/abl gene lacking abl exon a2 in a patient with chronic myeloid leukemia, Haematologica 84 (1999), 573-575.

[19] S. Iwata, S. Mizutani, S. Nakazawa and J. Yata, Heterogeneity of the breakpoint in the ABL gene in cases with BCR/ABL transcript lacking ABL exon a2, Leukemia 8 (1994), 1696-1702.

[20] J. Polak, Z. Zemanova, K. Michalova, H. Klamova, J. Cermak and C. Haskovec, A new case of chronic myeloid leukemia (CML) in myeloid blast crisis with an atypical (b3/a3) junction of the BCR/ABL gene [letter], Leukemia 12 (1998), 250.

[21] J. Cortes and H. Kantarjian, How I treat newly diagnosed chronic phase CML, Blood 120(7) (2012), 1390-1397.

[22] J.J.M. Dongen, E.A. Macintyre, J.A. Gabert, E. Delabesse, V. Rossi, G. Saglio, E. Gottardi, A. Rambaldi, G. Dotti, F. Griesinger, A. Parreira, P. Gameiro, M. González Diáz, M. Malec, A.W. Langerak, J.F. San Miguel and A. Biondi, Standardized RT-PCR analysis of fusion gene transcripts from chromosome aberrations in acute leukemia for detection of minimal residual disease Report of the BIOMED-1 Concerted Action: Investigation of minimal residual disease in acute leukemia, Leukemia 13 (1999), 1901-1928. 


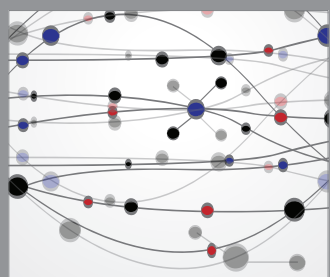

The Scientific World Journal
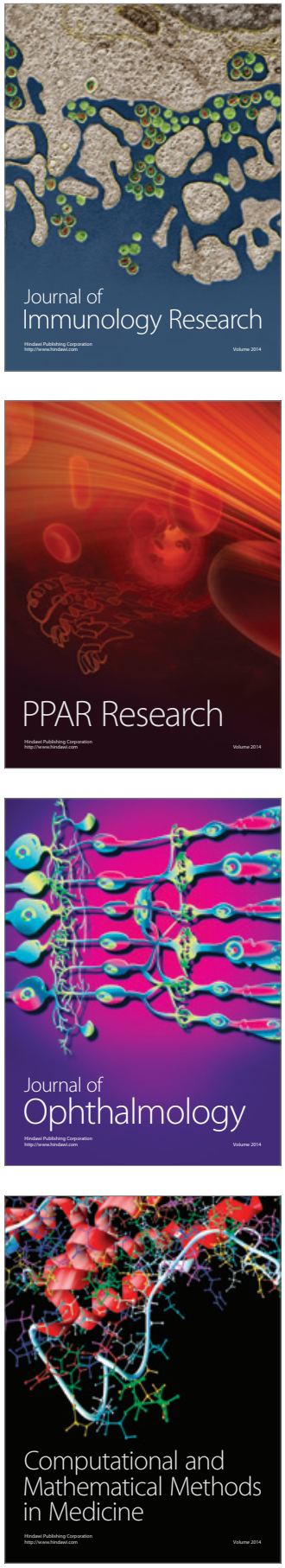

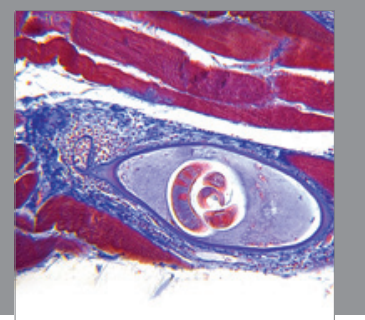

Gastroenterology

Research and Practice
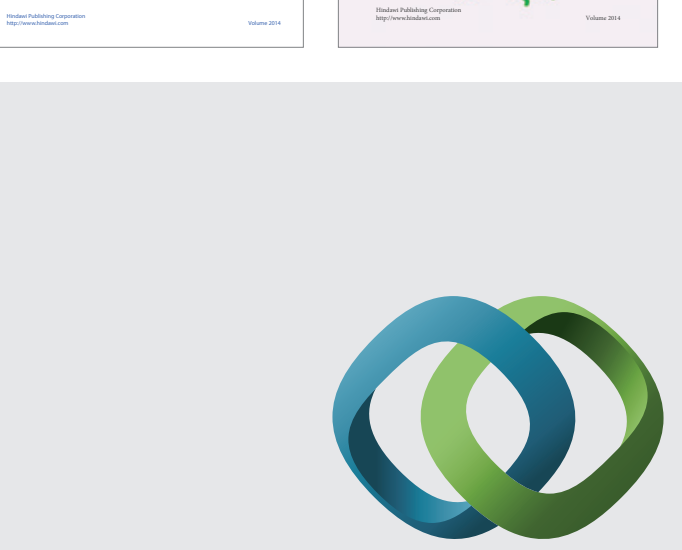

\section{Hindawi}

Submit your manuscripts at

http://www.hindawi.com
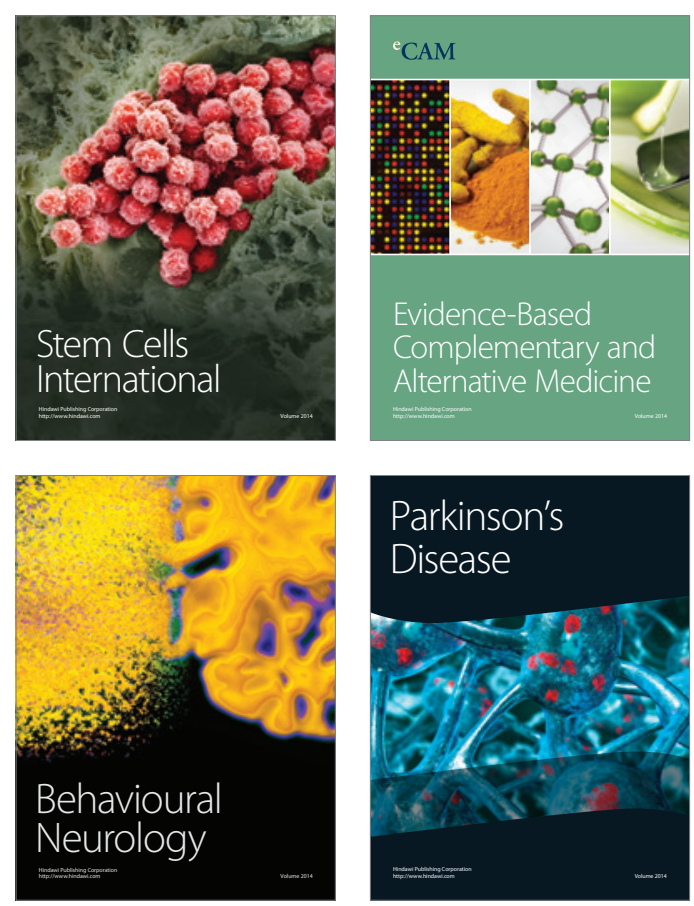

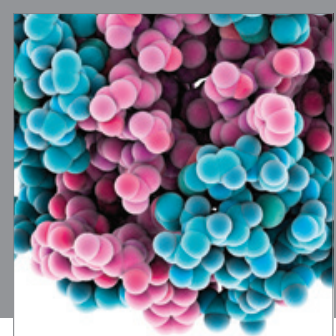

Journal of
Diabetes Research

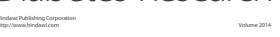

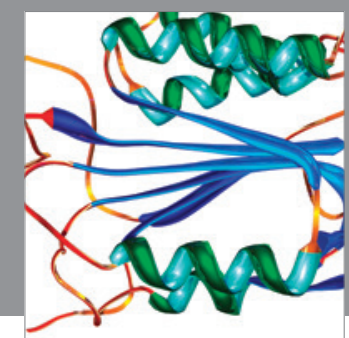

Disease Markers
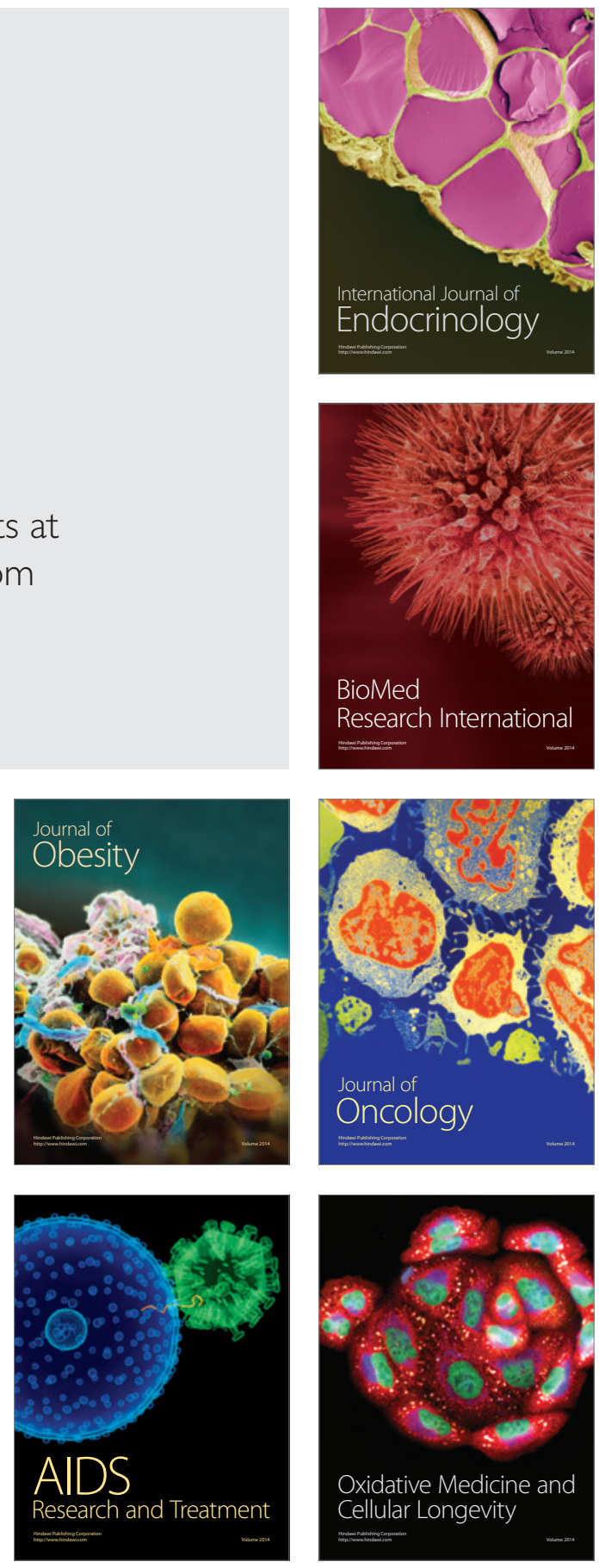